\title{
Study on Printer Characterization Based on BP Neural Network
}

\author{
Lei ZHAO \\ School of Media and Design, Hangzhou Dianzi University, Hangzhou, P.R.China
}

\begin{abstract}
There is a very complicated nonlinear relationship between the input digital image pixel value and the printed color chromatic values, which is difficult to be described by traditional linear methods to achieve high accuracy. In the study, a printer characterization model is established based on artificial BP neural network theory and by the hue angle range of the experimental data print classification. Because of the good simulation of nonlinear properties of the BP neural network, the characterization model achieves high accuracy. The mean color error value between the chromatic value to be printed and the chromatic value driven by the image pixel value which is calculated by the characterization model is rather less than that of the minimum range of the human eye can identify. The characterization model could be used in the gamut matching of the printing color management system, when the printer is severed as an output device.
\end{abstract}

KEYWORD: BP neural network; Characterization model; Hue angle range; Color printer

\section{INTRODUCTION}

The basic function of color printer is to print the corresponding color based on the value of the input digital image pixel. However, printers differ from each other in their working principles, structures and driver programs. Different printers may print different colors, when the same digital image pixel value is input. But for the same print, there is corresponding relationship between the two sets of data. In the study, the correspondence is considered as a black box, only the corresponding relationship between the output chromatic values and the input image pixel values is consider, regardless the color printing principles of the printer and the other factors. The color is described by CIE $1976 \mathrm{~L}^{*} \mathrm{a}^{*} \mathrm{~b}^{*}$ color system [1]. Printer characterization is to establish a model to describe the corresponding relationship between the printed color chromatic values $L^{*} a^{*} b^{*}$ and the digital image pixel values RGB [2-5], which could be used to calculate the input digital drive value based on the color to be printed on some paper by the printer. The characterization plays important roles in the printing color matching, graphic designing, especially in digital printing proofing.

Color printer prints colors by subtractive color principal. There is a very complicated nonlinear relationship between the input digital image pixel color values RGB and the printed colors chromatic values, which is described by linear method in the traditional characterization models [6]. The linear models achieved some results [7-14], but which cannot deal with the noise signal and other factor when the characterization is been carried out. In the study, a BP neural network with its nonlinear characteristics, self-learning and adaptive capacity is adopted, the experimental data is classified according to hue angle range, a color printer characterization model is established. In the printing color management system, printer characterization is a key link.

\section{DETERMINATION OF BP NEURAL NETWORK STRUCTURE}

In the study, there are 1000 groups of the experiment data to be collected, the number is rather large. The input data (color blocks CIE $1976 \mathrm{~L}^{*} \mathrm{a}^{*} \mathrm{~b}^{*}$ chromatic values) and output data (image pixel RGB value) scatter in the range of $[0,255]$ and $[0,100]$, $[-120,120], \quad[-120,120]$. A neural network with strong simulation ability is needed to transfer the experimental data samples with such features mentioned above. After repeated experiments, integrated with network approximation ability, training time and other factors, the structure of the neural network is determined. From the input layer to the output layer, the neurons number of the BP 
neural network is 3-30-20-30-3 respectively [15]. The network structure is shown as Figure 1.

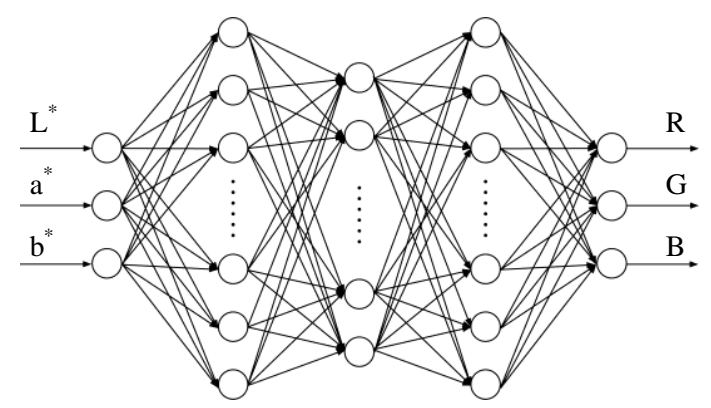

Figure 1. BP neural network

Among them, the input layer and output layer neurons are 3, the input layer correspond to the print color $\mathrm{L}^{*} \mathrm{a}^{*} \mathrm{~b}^{*}$ values, the 3 neurons of the output layer correspond to the corresponding digital image pixel value RGB, the number of the neurons of the 3 hidden layer are 30, 20,30. The activation functions of the hidden layer neurons are all Sigmoid functions [8], the activation function of the output layer neurons are all pure linear functions [16].

\section{ACQUIRING OF THE TRAINING SAMPLES OF BP NEURAL NETWORK}

Experiment color blocks are generated with selfgenerated programming based on the change of pixel values of a 24-bit true color BMP format digital image. Digital image pixel RGB values are selected at the 10 points of approximate the same intervals, the RGB values are $0,28,56,84,112,140,168$, $196,224,255$ respectively. 1000 groups of data pairs of digital image pixel values and its corresponding print color values are acquired based on the combination of the digital image pixel values in the 3 channels.

The 24-bit true color BMP image file that contains color blocks is printed on the printer, the type of the printer is EPSON Stylus Pro 7600. The printer is with high resolution and professional grade uses black, bright black, magenta, light goods, blue, bright blue, yellow 7-color ink, and a dedicated photo-printing on paper using printing blocks of color with a resolution of $300 \mathrm{dpi}$. The printer working condition became stable when it is turned on for 30 minutes. In the Photoshop CS6 without its color management modules activation, the color blocks are printed and measured with X-Rite Printer Optimizer one by one, the experimental data is recorded as Excel file via its supporting software Color Shop and the computer COM2 interface by data cable.

\section{TRAINING OF THE BP NEURAL NETWORK}

When the neural network is trained, it found that there has been a long time the objective function of the error convergence slow or stagnant under the supervision of the training samples. Combined with artificial neural networks theory and practical experience [17], it thinks that the training sample data that is of large number and more dispersed results in the condition mentioned above. In order to improve the training speed of neural network and achieve higher accuracy, according to the classification method on the training data with its brightness [18], the training data is classified with its hue angle range to facilitate the neural network training. The hue angle range of some training data is calculated as the following expression 1.

$$
\alpha=\tan ^{-1} \frac{b^{*}}{a^{*}}
$$

All the training data could be classified by its hue angle range in the CIE $1976 \mathrm{~L}^{*} \mathrm{a}^{*} \mathrm{~b}^{*}$ color space, and its corresponding digital images pixel value is recorded to form the data pair. The CIE $1976 \mathrm{~L} \mathrm{a}^{*} \mathrm{~b}^{*}$ color space is divided into 10 portions via $\mathrm{L}^{*}$ axis as the center, each portion is a hue angle range.

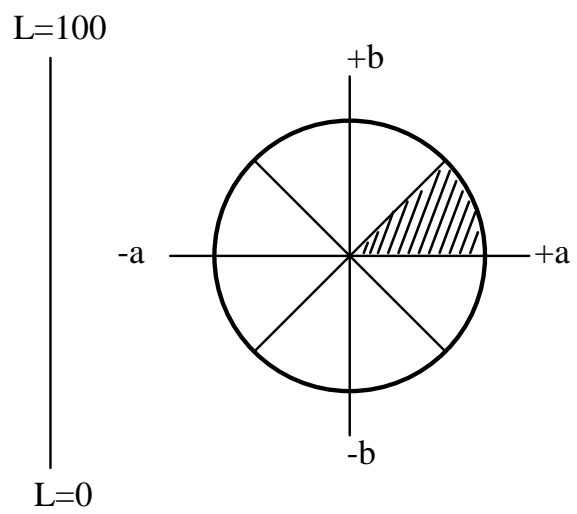

Figure 2. Hue angle range in CIE $1976 \mathrm{~L}^{*} \mathrm{a}^{*} \mathrm{~b}^{*}$ system

In Fig 2, the CIE $1976 \mathrm{~L}^{*} \mathrm{a}^{*} \mathrm{~b}^{*}$ color space is divided into 8 parts to $\mathrm{L}^{*}$ axis as the center, the slash part represents a hue angle range. Thus, the portion which is between the $2^{\text {nd }}$ hue angle ranges and the $8^{\text {th }}$ hue angle ranges is shown as the expression 2.

$$
[2 \pi(i / \text { SliNum })-T F, 2 \pi((i+1) / \text { SliNum })+T F]
$$

The expression above corresponds to the $(\mathrm{i}-1)^{\text {th }}$ hue angle range. Among the expression, SliceNumber(Abbreviated as SliNum) is the hue angle range number which the CIE $1976 \mathrm{~L}^{*} \mathrm{a}^{*} \mathrm{~b}^{*}$ color space divided into. TFactor(Abbreviated as TF) is the redundancy factor. In the study, TF is taken as $1 / 10$ of $2 \pi(1 /$ SliNum $)$. Thus, the expression of the $6^{\text {th }}$ hue angle range is as expression 3.

$$
[2 \pi(5 / \text { SliNum })-T F, 2 \pi(6 / \text { SliNum })+T F]
$$


The training data which locates in the hue angle range described in the expression above is used to train the $6^{\text {th }}$ neural network, so does in the other hue angle range and their corresponding training data. The expression of $1^{\text {st }}$ hue angle range and the $10^{\text {th }}$ hue angle range is shown as the expression 4 and 5, for that they locate at the edge of the whole hue angle ranges.

$$
[2 \pi-T F, 2 \pi] \cup[0,2 \pi(1 / \text { SliNum })+T F]
$$

$[2 \pi(9 /$ SliNum $)-T F, 2 \pi] \cup[0,2 \pi(1 /$ SliNum $)+T F](5)$

The introduction of redundant factors is to train network in a comparatively wider range and to realize the simulation function in the relatively narrow range to improve the network accuracy [19].

In this way, the printer characterization model consists of 10 sub-characterization models. When the characterization model is used to calculate the input digital image pixel value, the sequence number of the hue angle range where the color to be printed should be determined firstly, and then the RGB input digital image pixels value is calculated by its corresponding sub-characterization model. In the experiment, how the 1000 groups of training samples distribute according to their hue angle ranges in the CIE $1976 \mathrm{~L} \mathrm{a}^{*} \mathrm{~b}^{*}$ color space, and it is shown in Table 1.

Table 1. Distribution of the printer experimental data in each hue angle range.

\begin{tabular}{|c|c|c|}
\hline $\begin{array}{c}\text { Hue angle ranges } \\
\text { sequence }\end{array}$ & $\begin{array}{c}\text { Hue angle range } \\
\text { (unit: radian) }\end{array}$ & $\begin{array}{c}\text { Data number } \\
\text { in each hue } \\
\text { angle range }\end{array}$ \\
\hline 1 & $(6.220,2 \pi) \cup(0,0.691)$ & 75 \\
\hline 2 & $(0.565,1.319)$ & 101 \\
\hline 3 & $(1.194,1.948)$ & 131 \\
\hline 4 & $(1.822,2.576)$ & 231 \\
\hline 5 & $(2.450,3.204)$ & 97 \\
\hline 6 & $(3.079,3.833)$ & 58 \\
\hline 7 & $(3.707,4.461)$ & 53 \\
\hline 8 & $(4.335,5.089)$ & 87 \\
\hline 9 & $(4.964,5.718)$ & 216 \\
\hline 10 & $(0,0.063) \cup(5.592,2 \pi)$ & 134 \\
\hline
\end{tabular}

From Table 1, due to the introduction of redundant factors, the sum amount of data within the 10 hue angle ranges is 1183 , which is greater than the number of 1000 of training sample data that results from that part e data locate in two hue angle ranges at the same time.

To improve the training speed of neural networks, the training data is normalized to the range of $[-1,1]$ firstly [20]. Method of additional momentum factor and the combination of variable learning rate of neural network learning is adopted to train the neural network [21].

\section{RESULTS AND ANALYSIS}

81 groups of chromatic values of color blocks are selected randomly from the 1000 group of training data samples and input into the printer characterization model to calculate the corresponding digital image pixel values to be input. The color blocks with the pixel color values calculated by the printer characterization model are printed on photo-printing paper by the same printer and their chromatic values are measured and recorded.

The color errors between the two kinds of data are calculated in the CIE $1976 \mathrm{~L}^{*} \mathrm{a}^{*} \mathrm{~b} *$ with its color error formula, which is about the color of to-be printed on the printer driven by digital image pixel values calculated by printer characterization model and the actual color printed on the printer. The color error between the two kinds of data is shown in Figure 3 with their color blocks sequence number.

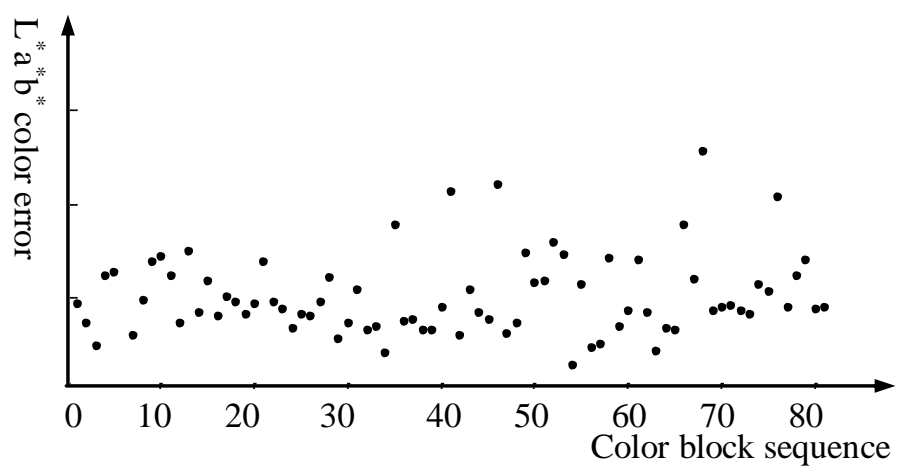

Figure 3. The distribution of color error between to be printed colors and actual display color

Thus, the colors to be printed on the printer and actual color printed on the printer is shown as the following. Amongst the parameters, there are average color error 2.135, maximum color error 9.125 and minimum color error 0.575 . The number of color error samples more than 6 is 1 .

Moreover, as it shows in Figure 3, the color errors concentrate in the number of 3 or less. Taken the factor that the printer repeatability error [24-28] is 0.719 , it indicates that the printer characterization model has a rather high conversion precision, and it can be applied in the color gamut matching.

\section{CONCLUSIONS}

In the study, based on BP neural network with improving combination of additional momentum factor and variable training speed3, a printer characterization model with experimental data classification according to hue angle ranges is proposed and established. Because of the good nonlinear approximation properties of BP network, the characterization model achieves rather high 
accuracy. The color error between the actual measured color chromatic values and the color driven by digital image pixel value calculated by printer characterization model is much less than the average minimum that the human eye can identify. Each sub-characterization model is established on the basis of experimental data classification by hue angle range, cooperated with gamut matching module, it could be used in the printing color management system with output of printer. The method that is about how to establish characterization model based on BP neural network can be used in the characterization model establishment of other devices such as scanner and even printer.

\section{ACKNOWLEDGMENTS}

This work is supported by the Research Project of the Zhejiang Province Education Department under Grant No. Y201223695.

\section{REFERENCES}

[1] S.S Zhou. 2013. Printing Color Science, Printing Industry Press, Beijing: China.

[2] G.W Hong, M.R Luo, P.A. Rhodes. 2001. A study of digital camera colorimetric characterization based on polynomial modeling, Color Research and Application, 26(1):76-84.

[3] D.Q. Zhu. 2013. Artificial Neural Networks and Its Applications, Science Press, Beijing: China.

[4] Q.M. Huang, D. Z Zhao. 2003. An evaluation method of different gamut mapping models, Journal of Beijing University of Technology, 23(1):601-603.

[5] V. Ostromoukhov, R. D. Herseh. 1994. Two approaches in scanner-printer characterization: colorimetric spacebased vs closed-loop, SPIE, 2170:133-142,

[6] K.L. Zhou, Y.H. Kang. 2012. Neural network model and simulation program design, Tsinghua University Press, Beijing: China.

[7] C.R. Yuan. 2013. Artificial neural networks and its application, Tsinghua University Press, Beijing: China.

[8] S.Q. Zhou, D.Z. Zhao. 2000. Study on liquid crystal display CD characterization, Optical Technology, 26(1):49-51.

[9] N. Katoh, T. Deguchi. 2001. An accurate characterization of CRT monitor verifications of past studies and clarifications of gamma, Optical Review, 8(5):305-314.
[10] D. L. Post, C. S. Calhoun. 1989. An evaluation of methods for producing desired colors on CRT monitors, Color Research and Application, 14(4):172-186.

[11] H. Kotera, A. Ishige, H. S. Chen. 1999. High precision scanner/printer calibration in subdivided color spaces, Journal of Imaging Science and Technology, 43(2):178186.

[12] W. Yang, N. Liao. 2003. Color reproduction on CRT displays via BP neural networks under office environment, Journal of Beijing Institute of Technology, 12(4):376-380.

[13] N. Liao, Z. Gao. 1999. A comparative study of a CRT colorimetric prediction model by neural networks and the models by conventional method, Color Research and Application, 24(1):45-51.

[14] N.F. Liao, J.S. Shi. 2009. Digital Image and Character Color Management System Sketch, Press of Beijing University of Technology, Beijing: China.

[15] S. K. Shevell. 2003. The science of color. Elsevier: England.

[16] Hunt. 2004. The reproduction of color. John Wiley \& Sons, Chichester: England.

[17] S. Cong. 2013. Toolbox for MATLAB Neural Network Theory and Application, China Science and Technology University Press, Hefei: China.

[18] K. Barnard, B. Funt. 2002. Camera characterization for color research. Color Research and Application, 27(3):152-163.

[19] D. L. Post, C. S. Calhoun. 1989. An evaluation of methods for producing desired colors on CRT monitors, Color Research and Application, 14(4):172-173.

[20] J. Thomas, J. Y. Hardeberg, I. Foucherot. 2008. The PLVC display color characterization model revisited, Color Research and Application, 33(6):449-460.

[21] E. A. Day, L. Taplin, R. S. Berns. 2004. Colorimetric characterization of a computer-controlled liquid crystal display, Color Research and Application, 29(5):365-373.

[22] G. Marcu, W. Chen. 2002. Color characterization issues for TFTLCD displays, SPIE, 4663:187-198.

[23] S. Wang, H.S. Xu. 2007. A LCD characterization S model algorithm, Letter of China Image and Graphic, 12(3):491494.

[24] S. Wen, R. Wu. 2006. Two-primary crosstalk model for characterizing liquid crystal displays, Color Research and Application, 31(2):102-108.

[25] Abrardoa, M.BarniL. 1999. CRT calibration for high quality reproduction of color images, SPIE, 3808: 52-59.

[26] Y. Wang, H. S. Xu. 2005. Determination of CRT gamut boundaries in perceptual color space, SPIE, 5637:332338.

[27] Cazes, F. Mintzer. 2001. Implementation of a calibration method for liquid crystal displays, SPIE, 4295:267-278.

[28] Bastani, B. Cressman, B. Funt. Funt. 2005. Calibrated color mapping between LCD and CRT displays: a case study, Color Research and Application, 30(6):438-447. 